\title{
Systematic study of the thermal properties of zeolitic frameworks
}

\author{
Maxime Ducamp and François-Xavier Coudert* \\ Chimie ParisTech, PSL Research University, CNRS, Institut de Recherche de Chimie \\ Paris, 75005 Paris, France \\ E-mail: fx.coudert@chimieparistech.psl.eu
}

\begin{abstract}
We report a systematic study of the thermal and mechanical properties of 134 pure $\mathrm{SiO}_{2}$ zeolites through DFT-based calculations by making use of the quasi-harmonic approximation (out of a total of 242 known fully ordered zeolitic frameworks). The comparison of our results with reported experimental data for several zeolites revealed a very good accuracy and validated our simulation methodology. We observe a wide range of thermal expansion coefficients (from -5 to $-35 \mathrm{MK}^{-1}$ ), highlighting the great influence of the framework topology over this physical property, while demonstrating that all pure-silica zeolites exhibit negative thermal expansion (NTE). Our simulations also provide a path for the computation of the bulk modulus for each structure, as well as its pressure and temperature dependence. Results revealed a large gamut of bulk modulus values (from 8 to $134 \mathrm{GPa}$ ), showing that most frameworks display pressure-induced softening - but not all! Finally, this study provides some hints to the open question of experimental feasibility of zeolitic frameworks, showing that the experimentally synthesized structures appear to have a distinct distribution of thermal and mechanical properties.
\end{abstract}




\section{Introduction}

Pure silica zeolites are nanoporous phases of $\mathrm{SiO}_{2},{ }^{1-3}$ that are metastable relative to the dense $\alpha$-quartz phase. They are also known as all-silica zeolites, siliceous zeolites, or zeosils. In addition to the usual applications of microporous materials as molecular sieves (for adsorption, fluid separation and gas storage) and heterogeneous catalysts, they have specific applications due to the hydrophobicity of their internal surface: the combination of small pore size and nonwetting surface can be leveraged to adsorb, store, or dissipate energy. ${ }^{4,5}$ They have also been proposed for practical applications in the form of thin films as low- $k$ materials, chemical sensors, membrane reactors, and microelectronic devices. ${ }^{6-8}$

The porous structure of zeolites find its root in their synthesis and crystallization in the presence of (typically organic) templating agents, before a calcination process that removes the template but retains the structural integrity, topological characteristics and porosity of the inorganic framework. Out of an mathematical infinity of four-connected zeolitic nets (due to the corner-sharing nature of the $\left[\mathrm{SiO}_{4}\right]$ tetrahedra), systematic exploration with classical interatomic potentials have shown that there are several hundred thousands of distinct lowenergy frameworks possible. ${ }^{9-11}$ Out of this large number, only 242 have been experimentally observed in natural or synthesized zeolitic materials, and less than a quarter of that number (46) can be experimentally obtained as pure silica compounds. While zeolites are amongst the most often used nanoporous materials at the industrial scale, these numbers highlight two very fundamental questions of zeolite science that are still widely open. First: what factors determine the viability, or experimental feasibility, of a given framework in a given chemical composition? ${ }^{12-14}$ It is known that energetic stability is not a sufficiently discriminating factor here, with many materials with low relative energy not being accessible by synthesis. ${ }^{10}$ Other criteria, such as entropy, mechanical stability, fit of the templating agent, etc. have been proposed but the question still remains mostly unanswered. ${ }^{15}$ Secondly: how can we establish structure-properties relationships within this large family of materials? On that front, there have been key advances for some properties, with large-scale computational predictions of 
adsorption, ${ }^{16}$ gas separation, ${ }^{17,18}$ and more recently mechanical properties. ${ }^{19,20}$ However, there are other properties that still escape our understanding, having not been studied in a systematic manner: diffusion and transport, thermal behavior, and most non-linear responses such as pressure and temperature-dependent physical properties.

In the present paper, we report a large-scale study of the thermal properties of synthesized and hypothetical pure silica zeolites, through quantum chemistry calculations at the density functional theory (DFT) level. After validating the accuracy of our proposed methodology against the scarce experimental data available, we perform calculations in the harmonic and quasi-harmonic approximations in order to compute both the thermal properties of zeolites, as well as the pressure and temperature dependence of their mechanical behavior. We show that there is a large impact of framework topology on these physical properties, opening the way for a better understanding of the structure-property relationships and providing highly-accurate ab initio data for the development of future models, either at the force field level or through machine learning techniques.

\section{Computational methods}

\section{Harmonic approximation}

Let us consider a crystal in mechanical equilibrium with Cartesian coordinates $R_{\kappa, \alpha}(\alpha$ representing the Cartesian $x, y, z$ directions), and with a unit cell containing $N$ atoms labelled $\kappa$. If $u_{\kappa, \alpha}$ represents the displacement of an atom $\kappa$ from its equilibrium position in the direction $\alpha$, the energy can be expressed as a Taylor expansion:

$$
E=E_{0}+\sum_{\kappa, \alpha}\left(\frac{\partial E}{\partial u_{\kappa, \alpha}}\right) u_{\kappa, \alpha}+\frac{1}{2} \sum_{\kappa, \kappa^{\prime}, \alpha, \alpha^{\prime}} H_{\alpha, \alpha^{\prime}}^{\kappa, \kappa^{\prime}} u_{\kappa, \alpha} u_{\kappa^{\prime}, \alpha^{\prime}}+\ldots
$$

where

$$
H_{\alpha, \alpha^{\prime}}^{\kappa, \kappa^{\prime}}=\left(\frac{\partial^{2} E}{\partial u_{\kappa, \alpha} \partial u_{\kappa^{\prime}, \alpha^{\prime}}}\right)
$$


are the second-order force constants, i.e., the elements of the Hessian matrix $H$. At equilibrium, residual forces are zero meaning that the first order term is null. The principle of the harmonic approximation is to neglect all anharmonic terms (corresponding to the third and higher order terms), keeping only the equilibrium lattice energy $E_{0}$ and the second-order term which corresponds to the harmonic energy.

For a periodic structure, we can write the displacements $u_{\kappa, \alpha}$ in terms of a plane wave with respect to the cell coordinates:

$$
u_{\kappa, \alpha}=\varepsilon_{m \kappa, \alpha q} e^{\left(i q \cdot R_{\kappa, \alpha}-\omega_{m} t\right)}
$$

With $q$ a phonon wave vector and $\varepsilon_{m \kappa, \alpha q}$ a polarization vector. This leads to an eigenvalue equation involving the dynamical matrix which corresponds to the mass-reduced Fourier transform of the Hessian matrix:

$$
D_{\alpha, \alpha^{\prime}}^{\kappa, \kappa^{\prime}}(q) \varepsilon_{m \kappa, \alpha q}=\omega_{m, q}^{2} \varepsilon_{m \kappa, \alpha q}
$$

With

$$
D_{\alpha, \alpha^{\prime}}^{\kappa, \kappa^{\prime}}=\frac{1}{\sqrt{M_{\kappa} M_{\kappa^{\prime}}}} \sum_{a} H_{\alpha, \alpha^{\prime}}^{\kappa, \kappa^{\prime}} e^{-i q \cdot r_{a}}
$$

This eigenvalue equation can be solved numerically, and the eigenvalues are obtained as the square of the vibrational frequencies. Second order force constants, which are needed to solve this equation, can be obtained through DFT calculations either by using the finitedisplacement method or the perturbation theory. ${ }^{21}$

While this approximation appears to be quite simple, its efficiency is no longer to be proven. ${ }^{22-24}$ The resulting mathematical model which admits exact solutions allows an accurate description of many features of the lattice dynamics such as the dependence of frequency on wave vector. However, despite being a reliable approximation for the description of lattice dynamics, it still shows some limitations. Indeed, under this approximation, vibrational 
frequencies are defined as independent of the interatomic distance meaning that the vibrational part of the energy is considered independent of the volume. Such a description leads to the failure of computing some thermodynamical properties: for example, thermal expansion would be equal to zero and thermal conductivity would be infinite due to the phonon lifetimes being infinite.

Nevertheless, one of the strengths of this approximation is that it can be easily modified to incorporate the missing anharmonic terms into a model that looks like a harmonic model.

\section{Quasi-harmonic approximation}

To overcome the weaknesses invoked previously, it is possible to introduce explicitly the anharmonic effects. This would require the calculation of phonon-phonon interactions that are missing in the harmonic approximation by, for example, computing higher order interatomic force constants or with the vibrational perturbation theory. ${ }^{25-27}$ Such techniques are computationally demanding and are not adapted to the scale of this study. Therefore, we chose to use the quasi-harmonic approximation which provides a simple correction for most of the previously mentioned drawbacks of the HA.

In the QHA, the Helmholtz free energy F can be described as:

$$
F(V, T)=U_{0}(V)-U_{v i b}(V, T)+T S
$$

Where $U_{0}$ is the zero-temperature lattice energy and $U_{v i b}$, the vibrational part of the energy, can be written as:

$$
U_{v i b}(V, T)=E_{0}(V) k_{B} T \sum_{k}\left(\ln \left(1-e^{\frac{\hbar \omega_{k}(V)}{k_{B} T}}\right)\right)
$$

Where $E_{0}(V)$ is the zero-point energy of the system, $k_{B}$ is the Boltzmann's constant, $\hbar$ is the Planck's constant and $\omega_{k}$ is the volume-dependent vibration frequency. Using 6 , the calculation of the thermodynamical properties such as Gibbs free energy, thermal expansion, 
bulk modulus or heat capacity can easily be achieved. As an example, Gibbs free energy can simply be obtained from the minimization of the following function with respect to the volume:

$$
G(P, T)=\min (F(V, T)+P V)
$$

From the volume-temperature relation $V(T)$ used to minimize $F(V, T)$, one can then determine the volumetric thermal expansion coefficient $\alpha_{V}$ as:

$$
\alpha_{V}(T)=\frac{1}{V(T)}\left(\frac{\partial V(T)}{\partial T}\right)_{P=0}
$$

\section{Grüneisen formalism}

Another useful formalism in this approximation is the Grüneisen parameter $\gamma$. This parameter has the advantage of linking directly the vibrational frequencies to the volume of the system, allowing then to study what effects the changes of temperature have on the crystal

lattice dynamics. For each vibration mode $i$ of the system, we can write a mode Grüneisen parameter as:

$$
\gamma_{i}=-\frac{V_{0}}{\omega_{i}\left(V_{0}\right)} \frac{\partial \omega_{i}(V)}{\partial V}
$$

An overall Grüneisen parameter can be obtained as the weighted average of all the mode Grüneisen parameter in terms of the corresponding contribution to the specific heat $C_{V, i}$ :

$$
\gamma(T)=\sum_{i} \frac{\gamma_{i} C_{V, i}(T)}{C_{V, i}(T)}
$$

Using this average Grüneisen parameter, the previously defined thermal expansion can also be expressed as:

$$
\alpha_{V}(T)=\frac{1}{V_{0} K_{T}} \sum_{i} \gamma_{i} C_{V, i}(T)
$$




\section{Mechanical properties and their pressure and temperature depen- dence}

In this section we list the methods used to obtain the mechanical properties described in this work.

Computation of the zero temperature bulk modulus $K_{0}$ and its derivative $K_{0}^{\prime}$ was realized through fitting of the volume/energy data with the third-order Birch-Murnaghan equation of state (EOS):

$$
E(V)=E_{0}+\frac{9 V_{0} K_{0}}{16}\left\{\left[\left({\frac{V_{0}}{V}}^{\frac{2}{3}}\right)-1\right]^{3} K_{0}^{\prime}+\left[\left({\frac{V_{0}}{V}}^{\frac{2}{3}}\right)-1\right]^{2}\left[6-4\left({\frac{V_{0}}{V}}^{\frac{2}{3}}\right)\right]\right\}
$$

Thermal effects on the bulk modulus were accounted for by making use of the QHA: The temperature dependent bulk modulus $K(T)$ was obtained as the isothermal second derivative of the free energy $F(V, T)$ with respect to the volume:

$$
K(T)=V(T)\left(\frac{\partial^{2} F(V, T)}{\partial V^{2}}\right)_{T}
$$

Such relation allowed the computation of the bulk modulus for each zeolite over the range of $10 \mathrm{~K}$ to $300 \mathrm{~K}$. Nevertheless, for the sake of clarity, we reported in this work only the values at $300 \mathrm{~K}$.

Finally, we characterize the temperature evolution of the bulk modulus $K(T)$ by defining a temperature coefficient $\delta$ as:

$$
\delta=\frac{1}{K(T)}\left(\frac{\mathrm{d} K(T)}{\mathrm{d} T}\right)
$$

\section{Calculation parameters}

All the simulations in this work were realized under the density functional theory as implemented in the CRYSTAL17 code. $^{28,29}$ All electron localized basis sets were used for both silicon and oxygen. They can be found on the CRYSTAL17 online library with the acronyms 
and references as follow: Si_88_31G*_nada_1996 ${ }^{30}$ for silicon and O_8_411_muscat_1999 ${ }^{31}$ for oxygen. Several functionals were tested for the description of the exchange and correlation term of the energy. We also tested the effect of Grimme-type dispersion corrections ${ }^{32}$ for each functional except PBESOL0 ${ }^{33}$ for which optimized coefficients are not currently available. However, this functional showed a better accuracy than the others even without considering the dispersion corrections. Therefore, we decided to choose PBESOL0 as the functional and did not use Grimme-type dispersion corrections in our calculations.

Mesh sampling was realized by using the Monkhorst-Pack scheme ${ }^{34}$ with a $k$-point mesh of $3 \times 3 \times 3$. For all the structures, supercells were generated in order to contain 150 atoms at most to ensure convergence of the thermal properties. Geometries were optimized using CRYSTAL17 code along with the default convergence criteria (a maximum of 0.00045 a.u. on atomic displacements during one optimization step and 0.0018 a.u. on forces). Representative input files and optimized structures are available online at https: //github.com/fxcoudert/citable-data.

Starting structures were taken from the International Zeolitic Association (IZA) database, considering only fully ordered frameworks, of which there are currently 242 known. Zeolites with unit cell containing more than 150 atoms were not considered, resulting in a set of 190 zeolites for which we optimized the geometry with a pure $\mathrm{SiO} 2$ composition. 134 structures were found to achieve convergence within the time constraints imposed. Quasi-harmonic approximation calculations were conducted with the QHA algorithm implemented in CRYSTAL1 $7^{35-38}$ over a volume deformation range of $-1.5 \%$ to $3 \%$ with 4 different volumes and thermal properties were computed over the range of $10 \mathrm{~K}$ to $300 \mathrm{~K}$. 


\section{Results and discussion}

\section{Validation of the methodology}

In order to validate our method and the various calculation parameters chosen (as described in the previous section), we selected 3 zeolitic structures for which experimental data are available: Linde type A (LTA framework), faujasite (FAU framework) and chabazite (CHA framework). We compare in this section our results, summarized in Table 1, with the experimental data from the literature. First, we relaxed the structures by optimizing both atomic positions and unit cell parameters. The unit cell parameters obtained are $12.014 \AA$ for LTA and $24.650 \AA$ for FAU (both cubic frameworks), with a difference of respectively $1.3 \%$ and $1.6 \%$ compared to the reported experimental values. The shifts are of the same order of magnitude for CHA (a trigonal crystal) with differences of $1.0 \%$ and $1.9 \%$ on the $a$ and $c$ parameters. These small differences can be ascribed to two factors: one is a possible thermal effect, as all calculated cell parameters are larger than experimental values, in line with a negative thermal expansion. A second effect is that our methodology uses a relatively small basis set - a more complete basis set could reduce the difference observed in the unit cell parameters. However, considering the number of calculations to be done in this study, such a choice would increase too much the overall computational cost and would not allow for our systematic study. Moreover, given that we are aiming at calculating thermal properties and the evolution of cell parameters with respect to temperature, we considered that these small discrepancies were acceptable.

We then conducted quasi-harmonic (QHA) calculations for these 3 systems and computed their thermal properties over the range of $10 \mathrm{~K}$ to $300 \mathrm{~K}$ - with values reported at $300 \mathrm{~K}$ in this section. We found that all of the structures show a negative thermal expansion (more on that later) and determined values of $-7.310^{-6} \mathrm{~K}^{-1}$ and $-4.910^{-6} \mathrm{~K}^{-1}$ for the linear thermal expansion coefficients of LTA and FAU, respectively, at $300 \mathrm{~K}$. These values are in very good agreement with the experimental measurements available: Carey et $\mathrm{al}^{39}$ obtained a 
Table 1: Lattice parameters and linear thermal expansion coefficients of pure silica zeolites with LTA, FAU, and CHA frameworks. Calculated values of thermal expansion coefficients are given at $300 \mathrm{~K}$.

\begin{tabular}{cccccc}
\hline & \multicolumn{2}{c}{ Lattice parameter $(\AA)$} & & Thermal expansion coefficient $\left(10^{-6} \mathrm{~K}^{-1}\right)$ \\
\cline { 2 - 3 } \cline { 5 - 6 } CODE & Expt. & Calc. & & Expt. & Calc. \\
\hline LTA & $11.853^{39}$ & 12.014 & & $-7.4^{39}$ & -7.3 \\
FAU & $24.256^{40}$ & 24.650 & & $-3.92^{40}$ & -4.9 \\
& & & & $-4.2^{41}$ & \\
CHA & $a=13.525^{42}$ & $a=13.671$ & & $-0.5^{42}$ & -0.6 \\
& $c=14.734^{42}$ & $c=15.015$ & & \\
\hline
\end{tabular}

mean value of $-7.410^{-6} \mathrm{~K}^{-1}$ for LTA between $100 \mathrm{~K}$ and $300 \mathrm{~K}$, very close to our calculated coefficient. As for the FAU framework, Leardini et al ${ }^{40}$ reported a value of $-3.9210^{-6} \mathrm{~K}^{-1}$ for a temperature range of $298-923 \mathrm{~K}$ while Attfield and Sleight ${ }^{41}$ obtained a coefficient of $-4.210^{-6} \mathrm{~K}^{-1}$ over a range of $25-573 \mathrm{~K}$. Even if the temperature range studied by Leardini et al. differs from ours, the reported values for both experiments agree reasonably well with our results. This is also the case for CHA, for which we calculated a linear thermal expansion coefficient of $-0.610^{-6} \mathrm{~K}^{-1}$, while Woodcock and Lightfoot ${ }^{42}$ obtained a value of $-0.510^{-6} \mathrm{~K}^{-1}$ at $293 \mathrm{~K}$.

Although there are relatively few experimental values reported in the literature for puresilica zeolites, the good quantitative agreement we found in these three different types of frameworks allowed us to validate our methodology and apply them to a large number of zeolitic frameworks.

\section{Volumetric thermal expansion coefficients}

We first turn our attention to a systematic assessment of the volumetric coefficients of thermal expansion of zeolitic frameworks. Within reasonable constraints on CPU time, we were able to obtain thermal expansion properties from QHA calculations for a total of 134 puresilica zeolites (see Figure 1). We present in Figure 2 the distribution of volumetric thermal expansion coefficients (on the $y$ axis). We see that the values range from -5 to $-32 \mathrm{MK}^{-1}$ 


\begin{tabular}{|c|c|c|c|c|c|c|c|c|c|c|c|c|c|c|}
\hline ABW & ACO & AEI & AEL & AEN & AET & AFG & AFI & AFN & AFO & AFR & AFS & AFT & AFV & AFX \\
\hline AFY & AHT & ANA & APC & APD & AST & ASV & ATN & АTO & ATS & ATT & ATV & AVE & AVL & AWO \\
\hline$A W W$ & BCT & BEC & BIK & BOF & BOG & BOZ & BPH & BRE & BSV & CAN & CAS & CDO & CFI & CGF \\
\hline CGS & CHA & $-\mathrm{CHI}$ & -CLO & CON & CSV & CZP & DAC & DDR & DFO & DFT & $\mathrm{DOH}$ & DON & EAB & EDI \\
\hline EEI & EMT & EON & EPI & ERI & ESV & ETL & ETR & ETV & EUO & EWO & EWS & EZT & FAR & FAU \\
\hline FER & FRA & GIS & GIU & GME & GON & GOO & HEU & IFO & IFR & -IFT & -IFU & IFW & IFY & IHW \\
\hline IMF & IRN & IRR & -IRY & ISV & ITE & ITG & ITH & ITR & ITT & -ITV & ITW & IWR & IWS & IWV \\
\hline IWW & JBW & JNT & JOZ & JRY & JSN & JSR & JST & JSW & KFI & LAU & LEV & LIO & -LIT & LOS \\
\hline LOV & LTA & LTF & LTJ & LTL & LTN & MAR & MAZ & MEI & MEL & MEP & MER & MFI & MFS & MON \\
\hline MOR & MOZ & MRT & MSE & MSO & MTF & MTN & MTT & MTW & MVY & MWF & MWW & NAB & NAT & NES \\
\hline NON & NPO & NPT & NSI & OBW & OFF & ОКО & OSI & oso & OWE & -PAR & PAU & PCR & PHI & PON \\
\hline POR & POS & PSI & PTT & PTY & PUN & PWN & PWO & PWW & RHO & - RON & RRO & RSN & RTE & RTH \\
\hline RUT & RWR & RWY & SAF & SAO & SAS & SAT & SAV & SBE & SBN & SBS & SBT & SEW & SFE & SFF \\
\hline SFG & SFH & SFN & SFO & SFS & SFW & SGT & SIV & SOD & SOF & SOR & sos & sov & SSF & SSY \\
\hline STF & STI & STT & STW & -SVR & sVV & SWY & $-S Y T$ & SZR & TER & THO & TOL & TON & TSC & TUN \\
\hline UEI & UFI & uos & uov & UOZ & USI & UTL & UWY & VET & VFI & VNI & vsv & WEI & -WEN & YFI \\
\hline YUG & ZON & & & & & & & & & & & & & \\
\hline
\end{tabular}

Figure 1: List of the 242 fully ordered zeolites from the IZA database. Red corresponds to synthesized structures, blue background corresponds to energy optimization and quasiharmonic approximation calculations realized in this work and green background corresponds to energy optimization done in this work.

(using for the sake of concision, the notation of $\mathrm{MK}^{-1}$ for $10^{-6} \mathrm{~K}^{-1}$ ). So we first see that all zeolitic frameworks feature negative thermal expansion (NTE), contrary to most materials that expand when heated. In the past, specific zeolites have been shown to display NTE, ${ }^{42-44}$ and the mechanism has been solidly established - at least qualitatively — as being due to the presence of rigid unit modes, ${ }^{45,46}$ arising from the corner-sharing tetrahedral nature of zeolite structures. ${ }^{47}$ Here, we show that this mechanism is actually entirely generic, and all zeolitic frameworks display NTE in their pure $\mathrm{SiO}_{2}$ form.

Given that the scientific literature contains a few examples of zeolites being reported with positive thermal expansion, ${ }^{48,49}$ this should prompt a re-examination of these materials: it is possible that such positive thermal expansion comes not from the zeolitic framework itself, but to the presence of trace of guest molecules, defects, or the occurrence of phase transitions in the temperature range under study.

The magnitude of the NTE displayed is on the same order of magnitude as some simple oxides and other porous materials, like AlPOs and metal-organic frameworks. ${ }^{50,51}$ While all zeolite frameworks are found to display NTE, we can also see on Figure 2 that the framework topology has an important impact on the thermal expansion of the crystalline structure even with fixed chemical composition, as is the case here. Previous computational studies, 

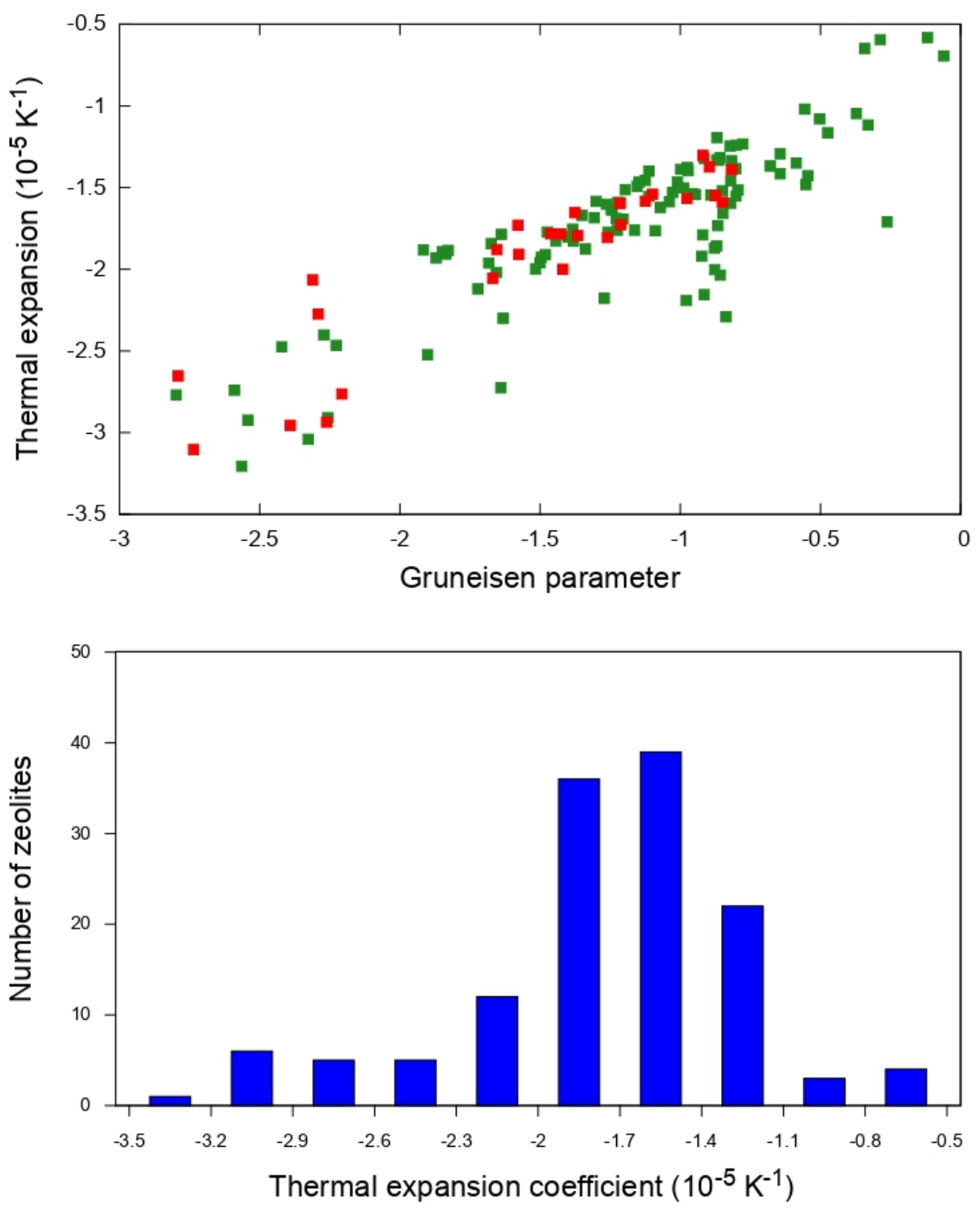

Figure 2: Top panel: Volumetric thermal expansion coefficients compared to the mean Grüneisen parameter for each zeolite at $300 \mathrm{~K}$. Green squares correspond to hypothetical zeolitic structures and red ones correspond to experimentally synthesized pure-silica frameworks. Bottom panel: Distribution of the values of thermal expansion coefficients for all the frameworks. 


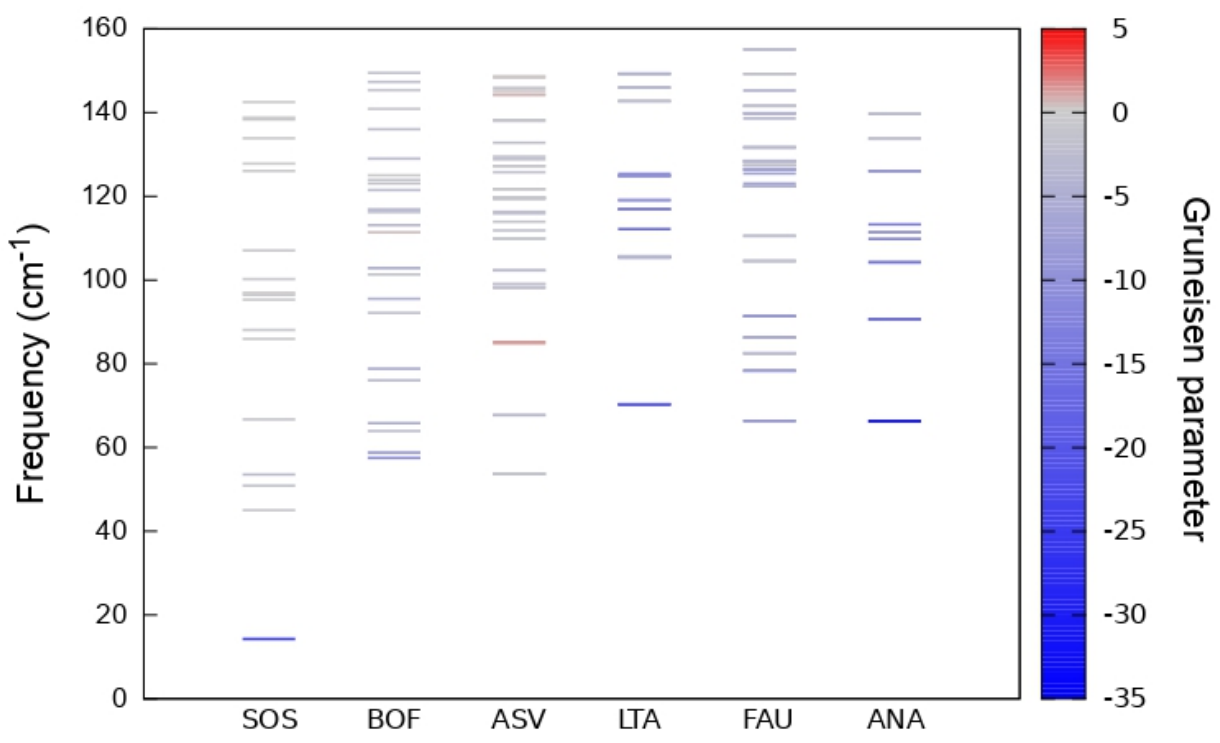

Figure 3: Representation of the mode Grüneisen parameter of 6 zeolites (SOS, BOF, ASV, LTA, FAU, ANA) for all the vibration modes under $160 \mathrm{~cm}^{-1}$. Each line represent a vibration mode (possibly degenerate due to symmetry), and its color indicates the value of the mode Grüneisen parameter.

through force-field based molecular dynamics, had drawn the conclusion that zeolites with only 1D channels tended not to show NTE behaviour, where as zeolites with 2D or 3D channels did. ${ }^{48,52,53}$ Our results here, obtained with a higher-accuracy methodology, do not confirm those trends. We have found no systematic and simple correlation between the characteristics of the zeolites (channel type, accessible pore volume, etc) and the value of the volumetric thermal expansion coefficient.

Moreover, we have not clearly identified a difference between zeolitic frameworks experimentally realized as pure-silica polymorphs, and other zeolitic frameworks (that have been obtained for chemical compositions other than $\mathrm{SiO}_{2}$ ) - those are represented in red and green respectively, on Figure 2. We do see that experimental structures cover almost the entire range, with perhaps a slight dominance over the high-NTE region, although that could be due to the relatively small size of our sampling of structures. This is in contrast with mechanical properties, where it was shown that experimental feasibility of the frameworks could be linked mechanical stability of the frameworks. ${ }^{19}$

Finally, we note that, on the temperature range studied here, the Grüneisen model for 
the study of the coupling of volume variations and vibrational properties ${ }^{54}$ is reasonably valid: Figure 2 displays the thermal expansion coefficients $\alpha_{V}$ against the mean Grüneisen parameter for each zeolite, and there is a broad correlation overall. In order to confirm the validity of the Grüneisen model of thermal expansion, we have looked at the individual lowfrequency vibration modes of different zeolites, 6 of which are depicted on Figure 3 . We can see, and have confirmed on another number of frameworks, that the negative mean Grüneisen parameter is crucially influenced by only a relatively small number of low-frequency vibrations modes, with strongly negative individual Grüneisen parameter. Those modes are found to be associated with frequencies below $130 \mathrm{~cm}^{-1}$, confirming the microscopic mechanism behind the NTE as being linked to soft vibration modes, involving concerted rotations of corner-sharing $\left[\mathrm{SiO}_{4}\right]$ tetrahedra.

\section{Anisotropy of thermal expansion}

In the previous section, we reported on the volumetric thermal expansion behavior of zeolites. Although several of the frameworks studied here have cubic symmetry (12 out of the 134 frameworks calculated), some zeolites have lower symmetry and anisotropy can be present. In particular, we identified structures in which some directions of space have positive linear thermal expansion — while others have a larger negative linear coefficient, contributing to the overall volumetric NTE. We depict in some detail the structure and thermal expansion profile of 3 such frameworks in Figure 4 - others can be found in the supporting information. In all three cases, the anisotropy can be clearly seen in the framework structure, and is giving rise to very modest positive coefficient along a single crystallographic axis. The number of such highly anisotropic materials is small (9 out of 134), although all non-cubic frameworks feature some level of anisotropy. It is interesting that the SOS framework type was previously demonstrated to have highly anisotropic mechanical properties, showing a link between thermal and mechanical properties through topology. ${ }^{19}$ 

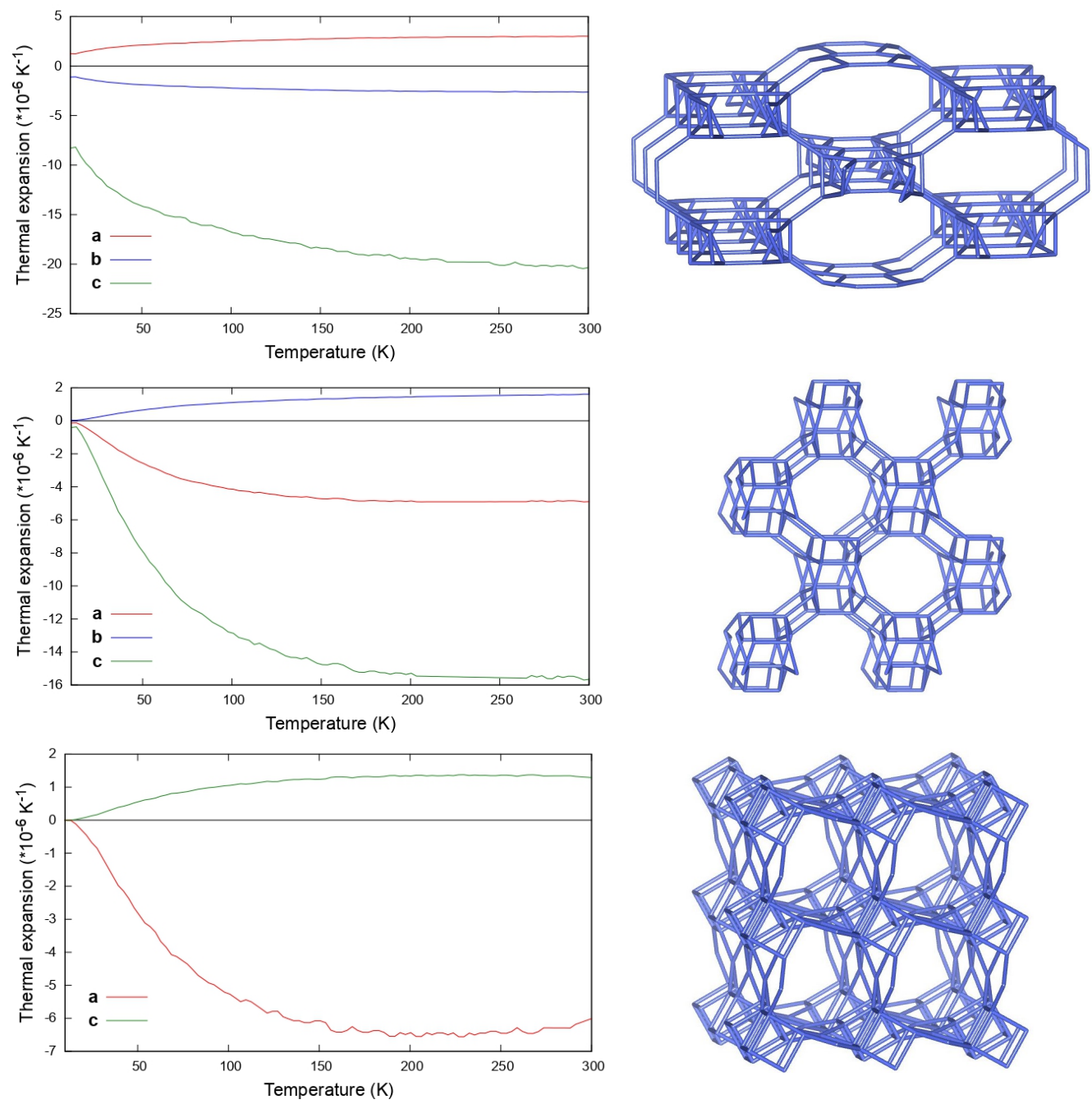

Figure 4: Evolution of the individual lattice parameters thermal expansion coefficients with respect to the temperature for the SOS, BOF, and ASV framework.

\section{Heat capacity and vibrational entropy}

From our calculations, we also gain access to phonon thermodynamics and physical quantities such as vibrational entropy of the crystal and heat capacity - they are displayed on Figure 5 and supporting information Figure S1. They offer a stark contrast with the large dependence on topology observed for thermal expansion, as they vary very little within the family of zeolitic frameworks. Vibrational entropy at $300 \mathrm{~K}$ is found to vary between 37 and $43 \mathrm{~J} \cdot \mathrm{mol}^{-1} \cdot \mathrm{K}^{-1}$ per $\mathrm{SiO}_{2}$ unit, with the majority of frameworks around $39 \mathrm{~J} \cdot \mathrm{mol}^{-1} \cdot \mathrm{K}^{-1}$. This is of relevance to the wider (and still pretty much open) question of relative stability 
and experimental feasibility of zeolites: ${ }^{12,55}$ how much does the entropic contribution affect the stability ordering of zeolitic frameworks? Based on the narrow distribution of $S$ values, the impact of entropy on relative free energy $(F=U-T S)$ is minimal: it is plotted as Figure S2. One can see that the free energy is dominated, at least at $300 \mathrm{~K}$, by the energetic contribution. At most, the contribution from vibrational entropy depending on framework topology will affect the relative stability by up to $\pm 3 \mathrm{~kJ} \mathrm{~mol}^{-1}$. This will be sufficient to affect the stability ordering of zeolitic frameworks only for those frameworks that are already energetically very close.

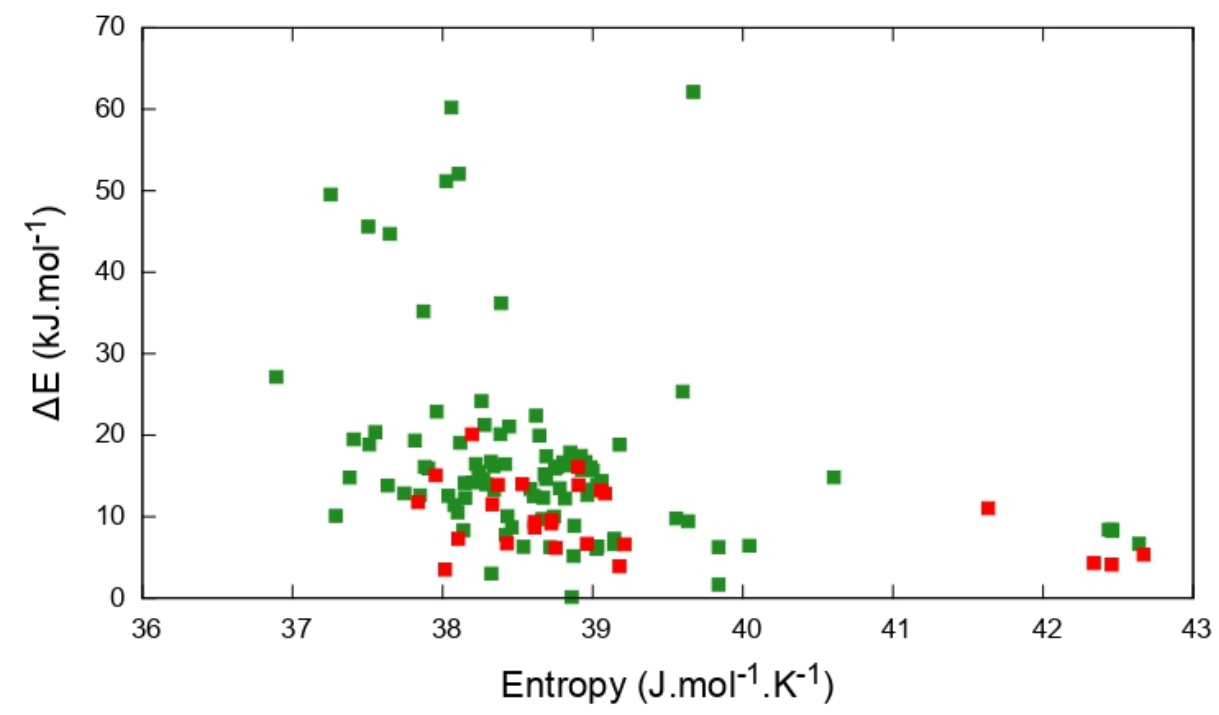

Figure 5: Energy relative to $\alpha$-quartz $(\Delta E)$ plotted against the vibrational entropy at $300 \mathrm{~K}$ $S_{\text {vib }}(300 K)$; both quantities are normalised per $\mathrm{SiO}_{2}$ unit. Green squares correspond to theoretical structures and red ones correspond to synthesized structures.

The distribution of heat capacity as a function of framework topology is every more narrow, with constant-volume heat capacity $C_{V}$ per $\mathrm{SiO}_{2}$ unit between 41.3 and $42.4 \mathrm{~J} . \mathrm{mol}^{-1} \cdot \mathrm{K}^{-1}$ at $300 \mathrm{~K}$. The influence of topology there is imperceptible, as for entropy, and in contrast with thermal expansion coefficients. This is explained by the link between the vibration modes and these physical properties: while thermal expansion is dominated by a small number of low-frequency modes, whose nature and frequencies are heavily dependent on the details of atomic arrangements, the entropy and heat capacity are averaged over all modes and 
therefore less sensitive to the topology of the framework, for a fixed chemical composition.

\section{Mechanical properties}

In previous computational studies, we have shown that the analysis of mechanical properties of inorganic framework materials in general, ${ }^{56}$ and zeolites in particular, ${ }^{19}$ could provide valuable insight into the relationship between structure and physical properties. These properties are typically calculated through strain-stress relationships, whether it is at the quantum chemical (DFT) level, ${ }^{19,57}$ using classical force fields, ${ }^{58}$ or through the application of machine learning methods. ${ }^{20,57}$ Whatever the accuracy of the interatomic description of the system, these mechanical properties were however systematically calculated in the linear elastic regime at the zero Kelvin limit. ${ }^{59,60}$ In particular, the temperature and pressure dependence of the elastic coefficients or other derived mechanical properties (elastic moduli, Poisson's ratio, etc.) have not been systematically studied. There is no data available in the literature on the range of values of those pressure and temperature dependence, nor on the impact of framework topology in a given class of materials.

Through the use of quasi-harmonic calculations, we were able to characterize for the first time not only the bulk modulus for each zeolitic structure, but also its pressure and temperature dependencies. We first present the distribution of bulk modulus values $K$ in Figure 6 : the average bulk modulus is $\sim 78.5 \mathrm{GPa}$, and the distribution range is $\sim 126 \mathrm{GPa}$ (from 8 to $134 \mathrm{GPa}$ ). There is therefore a clear effect of framework topology, with a change of one order of magnitude between the softest and stiffest frameworks. In addition, by plotting the data against the relative polymorph energy $\Delta E$ (top panel of Figure 6) we observed two interesting effects: first, a weak correlation between $K$ and $\Delta E$, meaning that the less stable zeolitic frameworks also tend to be mechanically softer - an effect that is also linked to the

density of the frameworks, known to be correlated with relative energy (see Figure S3). A second effect is a difference in behavior between experimentally known frameworks (in the $\mathrm{SiO}_{2}$ composition) and others: it is clear that most experimentally feasible pure-silica zeolites 

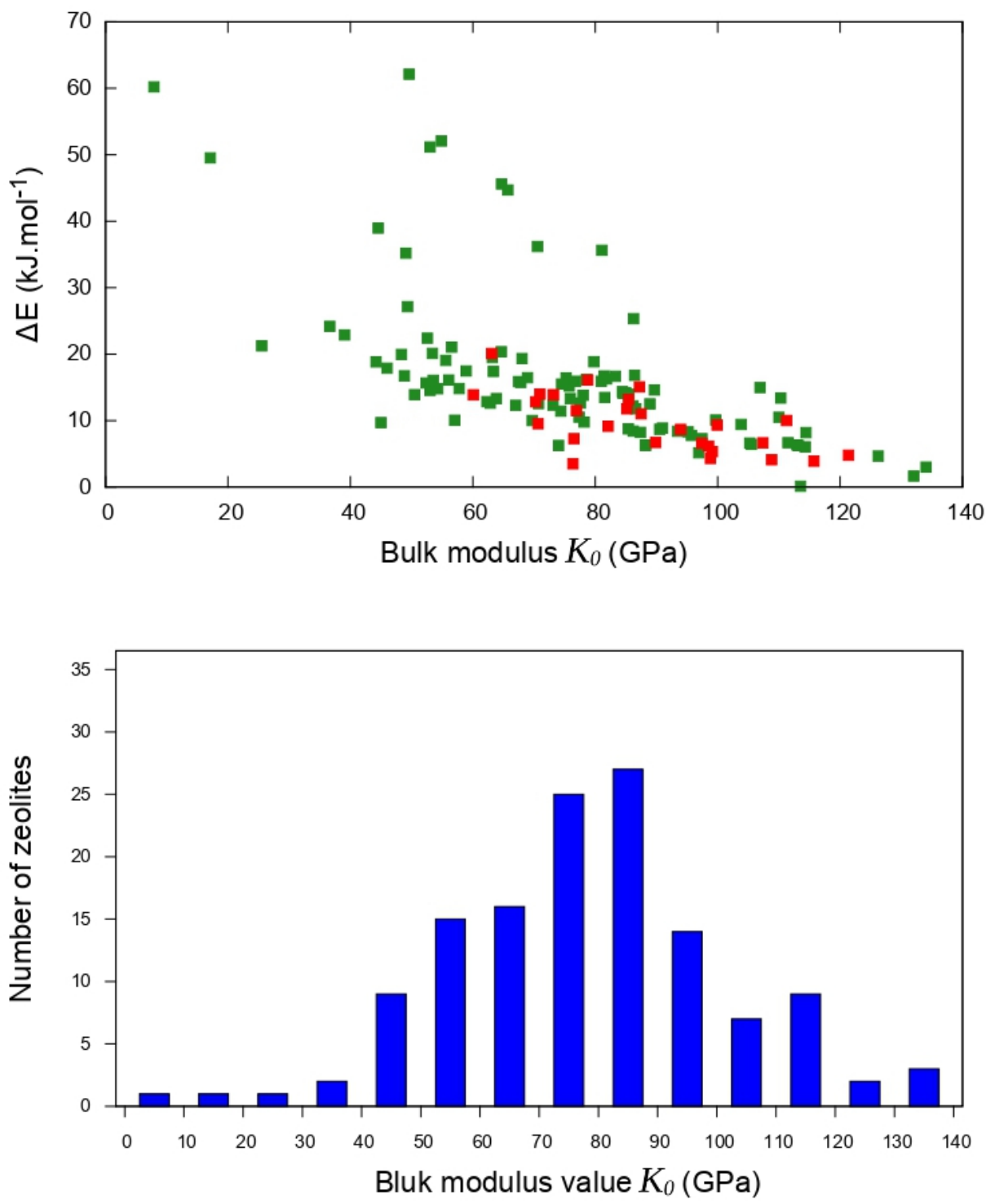

Figure 6: Top panel: Energy relative to $\alpha$-quartz $(\Delta E)$ plotted against the bulk modulus $K_{0}$ obtained through the Birch-Murnaghan equation of state. Green squares correspond to theoretical structures and red ones correspond to synthesized structures. Bottom panel: distribution of the $K_{0}$ values for all the frameworks. 

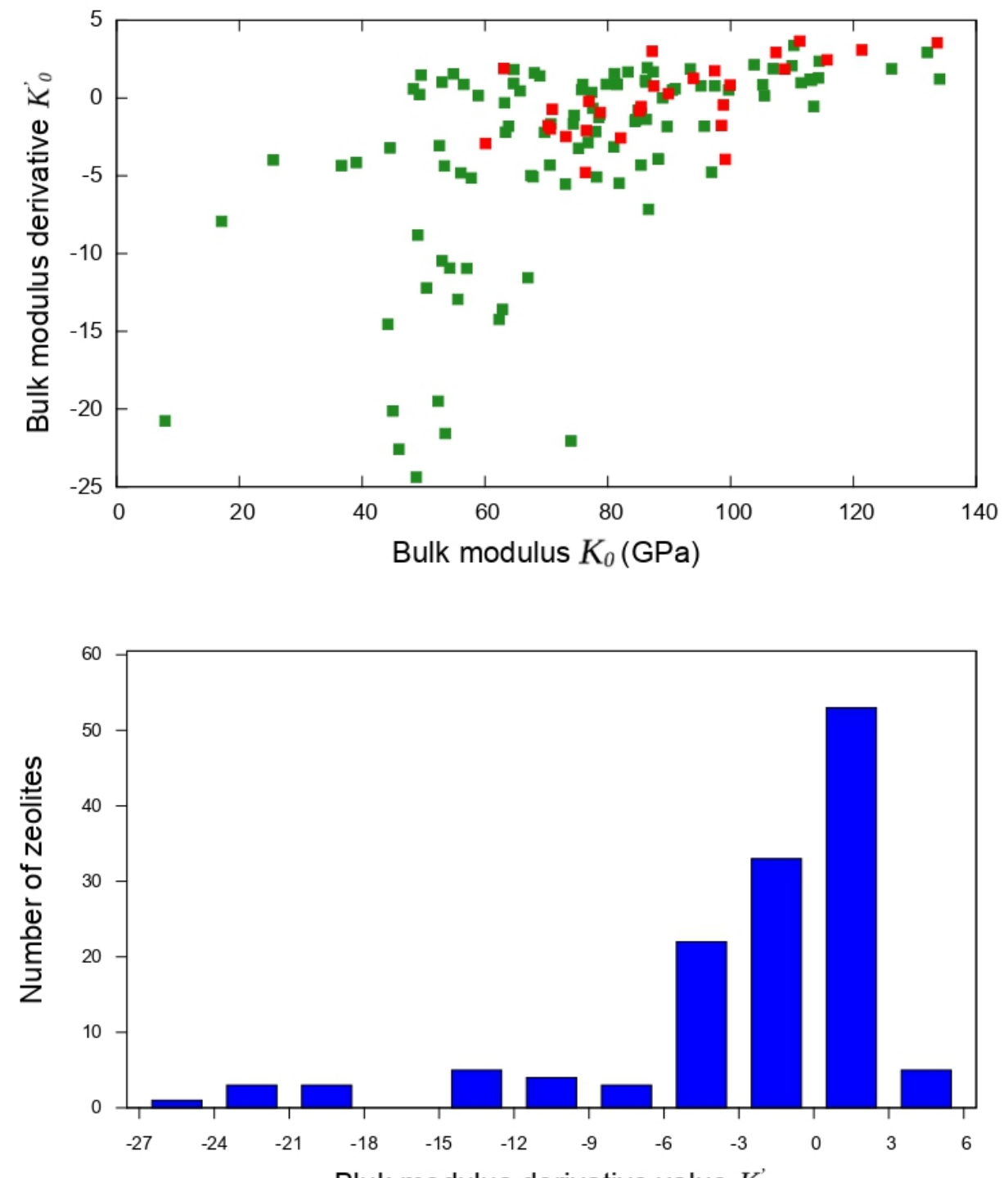

Bluk modulus derivative value $K_{0}^{\prime}$

Figure 7: Top panel: Bulk modulus $K_{0}$ plotted against its pressure-derivative $K_{0}^{\prime}$ for each zeolite. Green squares correspond to theoretical structures and red ones correspond to synthesized structures. Bottom panel: Distribution of the values of $K_{0}^{\prime}$ for all the frameworks. 
are among those with highest bulk modulus.

We also have access, for the first time in a systematical way across the zeolite family, to derivatives of the bulk modulus with respect to pressure (Birch-Murnaghan $K_{0}^{\prime}$ coefficient) and temperature (which we denote $\delta=(1 / K)(\partial K / \partial T))$. We see on Figure 7 that there is a wide impact of framework topology on $K_{0}^{\prime}$, showing a range of values both negative and positive, from -25 to +5 ( $K_{0}^{\prime}$ is dimensionless). We see that many zeolites feature negative $K_{0}^{\prime}$, featuring an abnormal softening of the elastic modulus with compression that can be linked to the mechanism already established for pressure-induced amorphization of zeolitic frameworks. ${ }^{61,62}$ Such a pressure-induced behavior has been previously identified in a small number of framework materials (including porous inorganic compounds). ${ }^{63-65}$ It has even been suggested that pressure-induced softening could be generally linked to negative thermal expansion, ${ }^{66,67}$ although we show here that it is not direct and systematic link: all $\mathrm{SiO}_{2}$ frameworks studied in this work show NTE, but only $56 \%$ exhibit pressure-induced softening (and the vast majority of structures are within the -5 to +3 range.

We note, in addition, that among the frameworks with large negative values of $K_{0}^{\prime}$, there are only structures that have not been synthesized in the $\mathrm{SiO}_{2}$ form. We infer from, as well as the conclusions on $K_{0}$ above, a confirmation of the previously suggested mechanical criterion to the experimental feasibility of zeolites.

Finally, we also investigated the temperature-dependence $\delta$ of the bulk moduli of the zeolitic frameworks. Contrary to the pressure-dependence, we see (Figure 8) that the impact of temperature is relatively small, making no marked change at $300 \mathrm{~K}$ to the overall values of bulk modulus, compared to $T=0$ (Figure S7). Moreover, we observe that the effect of temperature always goes in the direction of a softening upon heating (all values of $\delta$ significantly different from zero, within our accuracy, are negative). We note that most values are in the range of zero to $-10^{-3} \mathrm{~K}^{-1}$, with only a small number of exceptions - among which we find both theoretical and experimental $\mathrm{SiO}_{2}$ frameworks. 


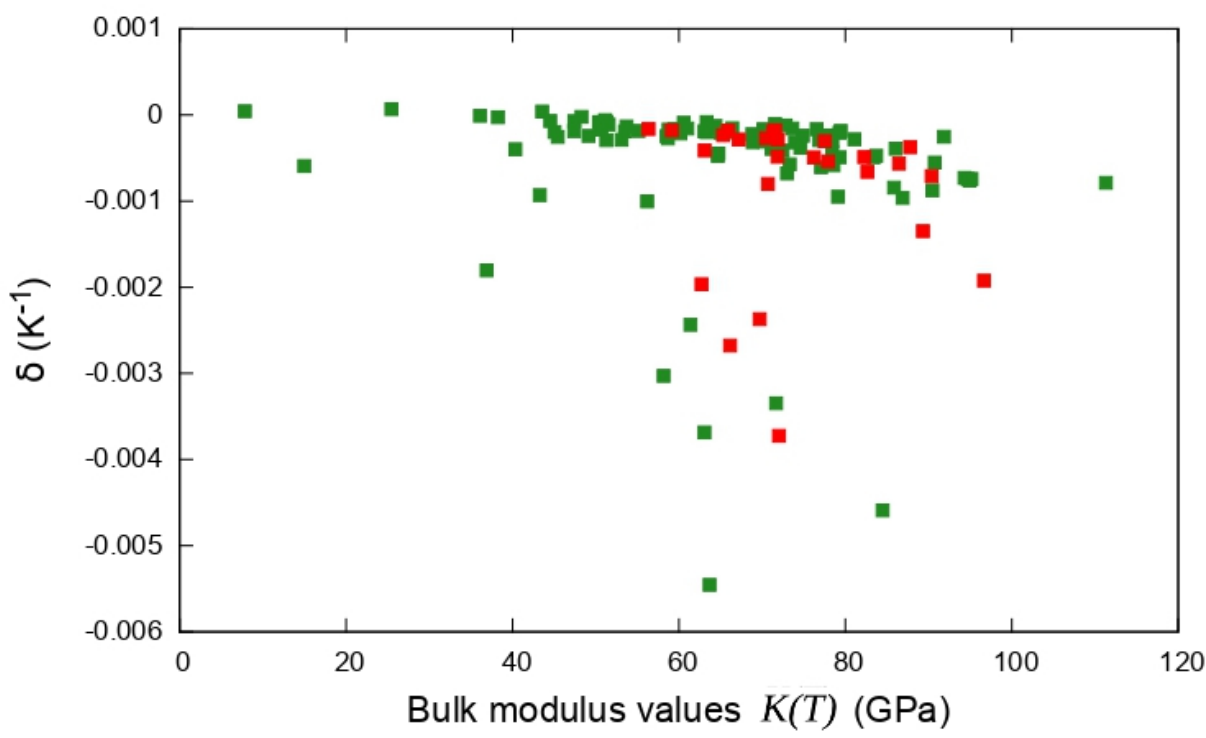

Figure 8: Temperature-dependent bulk modulus $K(T)$ compared to the temperature coefficient of elastic stiffness $\delta$ for all frameworks. Green squares correspond to theoretical structures and red ones correspond to synthesized structures.

\section{Conclusions and perspectives}

By a systematic study of 134 pure $\mathrm{SiO}_{2}$ zeolites through quasi-harmonic calculations at the density functional theory (DFT) level, we have been able to characterize generic trends in the thermal and thermo-mechanical properties of zeolitic frameworks. we first validated the quasi-harmonic approximation through comparison with experimental data for several zeolites, demonstrating a very good accuracy for our simulation methodology. We observed a wide range of thermal expansion coefficients for zeolites (from -5 to $-35 \mathrm{MK}^{-1}$ ), highlighting the important role of framework topology on thermal properties. We demonstrated, however, that all pure-silica zeolites exhibit negative thermal expansion (NTE), highlighting the systematic nature of this phenomenon in these four-connected nets. We could also probe the pressure and temperature dependence of the materials' bulk modulus, revealing a large gamut of bulk modulus values (from 8 to $134 \mathrm{GPa}$ ) and showing that most frameworks display pressure-induced softening. While this systematic study offers important new information on the range of possible thermal and thermo-mechanical behavior amongst zeolitic frameworks, we intend in the future to dig further into the microscopic details of these fea- 
tures, and use tools such as rigid-unit mode (RUM) analysis in order to identify the roots of such anomalous behaviors as pressure-induced softening.

This study also provides some more hints to the open question of experimental feasibility of zeolitic frameworks, showing that the experimentally synthesized structures display a distinct distribution of thermal and mechanical properties. While this does not solve the question, it does reinforce the idea that thermo-mechanical properties are a part of the criteria necessary to describe the feasibility of structures ${ }^{19}$ in addition to more commonly considered criteria such as energetic stability, entropic stabilization, solvation energies, and template effects. This can be understood if we interpret it this way: for a structure to be sufficiently stable for experimental synthesis, it needs to be a local energy minimum sufficiently deep that it can resist various stresses during synthesis and activation.

Within this work, we have generated dataset of $a b$ initio information of relatively large size and scope, on a chemically homogeneous family of materials. Due to the nature of the calculations, this is more computationally costly than previous similar systematic studies focused on other physical properties, like elastic properties. But this also means that we now have access to more information about the behavior of the system, in the form of the Hessian matrices for both the relaxed structures but also strained frameworks with different values of volume. This data can be useful for future exploration and as a basis of high-accuracy quantum chemistry data for benchmarking other methods and developing new models. In particular, it could be used as a training database for a machine learning study of thermal and thermo-mechanical properties based on structural features. ${ }^{20,57}$ Another possible use of this ab initio data would be to improve upon the currently available force fields for zeolites, ${ }^{68-70}$ by use for force field fitting techniques based on accurate quantum chemical reference data. ${ }^{71-73}$ 


\section{Acknowledgement}

We acknowledge financial support from the Agence Nationale de la Recherche under project "MATAREB" (ANR-18-CE29-0009-01) and access to high-performance computing platforms provided by GENCI grant A0090807069.

\section{Supporting Information Available}

Optimized structures, full tables of the thermal and mechanical properties and representative input files for the calculations are available online at https://github.com/fxcoudert/ citable-data

\section{References}

(1) S. M. Auerbach; K. A. Carrado; P. K. Dutta; Handbook of Zeolite Science and Technology, CRC Press, 2003.

(2) Astala, R.; Auerbach, S. M.; Monson, P. A. Density Functional Theory Study of Silica Zeolite Structures: Stabilities and Mechanical Properties of SOD, LTA, CHA, MOR, and MFI. J. Phys. Chem. B 2004, 108, 9208-9215.

(3) Piccione, P. M.; Laberty, C.; Yang, S.; Camblor, M. A.; Navrotsky, A.; Davis, M. E. Thermochemistry of Pure-Silica Zeolites. J. Phys. Chem. B 2000, 104, 10001-10011.

(4) Eroshenko, V.; Regis, R.-C.; Soulard, M.; Patarin, J. Energetics: A New Field of Applications for Hydrophobic Zeolites. J. Am. Chem. Soc. 2001, 123, 8129-8130.

(5) Fraux, G.; Coudert, F.-X.; Boutin, A.; Fuchs, A. H. Forced intrusion of water and aqueous solutions in microporous materials: from fundamental thermodynamics to energy storage devices. Chem. Soc. Rev. 2017, 46, 7421-7437. 
(6) Davis, M. E. Ordered porous materials for emerging applications. Nature 2002, 417, $813-821$.

(7) McLeary, E.; Jansen, J.; Kapteijn, F. Zeolite based films, membranes and membrane reactors: Progress and prospects. Micropor. Mesopor. Mater. 2006, 90, 198-220.

(8) Caro, J.; Noack, M. Zeolite membranes - Recent developments and progress. Micropor. Mesopor. Mater. 2008, 115, 215-233.

(9) Earl, D. J.; Deem, M. W. Toward a Database of Hypothetical Zeolite Structures. Ing. Eng. Chem. Res. 2006, 45, 5449-5454.

(10) Pophale, R.; Cheeseman, P. A.; Deem, M. W. A database of new zeolite-like materials. Phys. Chem. Chem. Phys. 2011, 13, 12407.

(11) Helfrecht, B. A.; Semino, R.; Pireddu, G.; Auerbach, S. M.; Ceriotti, M. A new kind of atlas of zeolite building blocks. J. Chem. Phys. 2019, 151, 154112.

(12) Bushuev, Y. G.; Sastre, G. Feasibility of Pure Silica Zeolites. J. Phys. Chem. C 2010, $114,19157-19168$.

(13) Sastre, G.; Corma, A. Predicting Structural Feasibility of Silica and Germania Zeolites. J. Phys. Chem. C 2010, 114, 1667-1673.

(14) Liu, X.; Valero, S.; Argente, E.; Botti, V.; Sastre, G. The importance of T ... T ... T angles in the feasibility of zeolites. Z. Kristallogr. Cryst. Mater. 2015, 230, 27.

(15) Li, Y.; Yu, J. New Stories of Zeolite Structures: Their Descriptions, Determinations, Predictions, and Evaluations. Chem. Rev. 2014, 114, 7268-7316.

(16) Krishna, R.; van Baten, J. M. In silico screening of zeolite membranes for $\mathrm{CO}_{2}$ capture. J. Membrane Sci. 2010, 360, 323-333. 
(17) Kim, J.; Abouelnasr, M.; Lin, L.-C.; Smit, B. Large-Scale Screening of Zeolite Structures for $\mathrm{CO}_{2}$ Membrane Separations. J. Am. Chem. Soc. 2013, 135, 7545-7552.

(18) Matito-Martos, I.; Martin-Calvo, A.; Gutiérrez-Sevillano, J. J.; Haranczyk, M.; Doblare, M.; Parra, J. B.; Ania, C. O.; Calero, S. Zeolite screening for the separation of gas mixtures containing $\mathrm{SO}_{2}, \mathrm{CO}_{2}$ and CO. Phys. Chem. Chem. Phys. 2014, 16, 19884.

(19) Coudert, F.-X. Systematic investigation of the mechanical properties of pure silica zeolites: stiffness, anisotropy, and negative linear compressibility. Phys. Chem. Chem. Phys. 2013, 15, 16012.

(20) Evans, J. D.; Coudert, F.-X. Predicting the Mechanical Properties of Zeolite Frameworks by Machine Learning. Chem. Mater. 2017, 29, 7833-7839.

(21) Görling, A.; Levy, M. Exact Kohn-Sham scheme based on perturbation theory. Phys. Rev. A 1994, 50, 196-204.

(22) Pascale, F.; Zicovich-Wilson, C. M.; Orlando, R.; Roetti, C.; Ugliengo, P.; Dovesi, R. Vibration Frequencies of $\mathrm{Mg}_{3} \mathrm{Al}_{2} \mathrm{Si}_{3} \mathrm{O}_{12}$ Pyrope. An ab Initio Study with the CRYSTAL Code. J. Phys. Chem. B 2005, 109, 6146-6152.

(23) Carteret, C.; Pierre, M. D. L.; Dossot, M.; Pascale, F.; Erba, A.; Dovesi, R. The vibrational spectrum of CaCO3aragonite: A combined experimental and quantummechanical investigation. J. Chem. Phys. 2013, 138, 014201.

(24) Erba, A.; Casassa, S.; Dovesi, R.; Maschio, L.; Pisani, C. Periodic density functional theory and local-MP2 study of the librational modes of Ice XI. J. Chem. Phys. 2009, 130, 074505 .

(25) Adel, A.; Dennison, D. M. The Infrared Spectrum of Carbon Dioxide. Part I. Phys. Rev. 1933, 43, 716-723. 
(26) Wilson, E. B.; Howard, J. B. The Vibration-Rotation Energy Levels of Polyatomic Molecules I. Mathematical Theory of Semirigid Asymmetrical Top Molecules. J. Chem. Phys. 1936, 4, 260-268.

(27) Nielsen, H. H. The Vibration-Rotation Energies of Polyatomic Molecules. Phys. Rev. 1941, 60, 794-810.

(28) Dovesi, R.; Erba, A.; Orlando, R.; Zicovich-Wilson, C. M.; Civalleri, B.; Maschio, L.; Rérat, M.; Casassa, S.; Baima, J.; Salustro, S.; Kirtman, B. Quantum-mechanical condensed matter simulations with CRYSTAL. Wiley Interdiscip. Rev. Comput. Mol. Sci. 2018, 8, e1360.

(29) Dovesi, R.;Saunders, V. R.;Roetti, C.;Orlando, R.;Zicovich-Wilson, C. M.;Pascale, F.;Civalleri, B.;Doll, K.;Harrison, N. M.;Bush, I. J.;D’Arco, P.;Llunell, M.; Causà, M.; Noël, Y.; Maschio, L.; Erba, A.; Rerat, M.; Casassa, S. CRYSTAL17 User's Manual. University of Torino: Torino, 2017.

(30) Nada, R.; Nicholas, J. B.; McCarthy, M. I.; Hess, A. C. Basis sets for ab initio periodic Hartree-Fock studies of zeolite/adsorbate interactions: $\mathrm{He}, \mathrm{Ne}$, and Ar in silica sodalite. Int. J. Quantum Chem. 1996, 60, 809-820.

(31) J. Muscat, 1999, "The phase stability, surface structure and defect chemistry of titanium dioxide from first principles techniques", PhD Thesis, University of Manchester, Manchester.

(32) Grimme, S. Semiempirical GGA-type density functional constructed with a long-range dispersion correction. J. Comput. Chem. 2006, 27, 1787-1799.

(33) Perdew, J. P.; Ruzsinszky, A.; Csonka, G. I.; Vydrov, O. A.; Scuseria, G. E.; Constantin, L. A.; Zhou, X.; Burke, K. Restoring the Density-Gradient Expansion for Exchange in Solids and Surfaces. Phys. Rev. Lett. 2008, 100. 
(34) Monkhorst, H. J.; Pack, J. D. Special points for Brillouin-zone integrations. Phys. Rev. B 1976, 13, 5188-5192.

(35) Erba, A. On combining temperature and pressure effects on structural properties of crystals with standardab initiotechniques. J. Chem. Phys. 2014, 141, 124115.

(36) Erba, A.; Shahrokhi, M.; Moradian, R.; Dovesi, R. On how differently the quasiharmonic approximation works for two isostructural crystals: Thermal properties of periclase and lime. J. Chem. Phys. 2015, 142, 044114.

(37) Erba, A.; Maul, J.; Pierre, M. D. L.; Dovesi, R. Structural and elastic anisotropy of crystals at high pressures and temperatures from quantum mechanical methods: The case of $\mathrm{Mg}_{2} \mathrm{SiO}_{4}$ forsterite. J. Chem. Phys. 2015, 142, 204502.

(38) Erba, A.; Maul, J.; Demichelis, R.; Dovesi, R. Assessing thermochemical properties of materials through ab initio quantum-mechanical methods: the case of $\alpha-\mathrm{Al}_{2} \mathrm{O}_{3}$. Phys. Chem. Chem. Phys. 2015, 17, 11670-11677.

(39) Carey, T.; Tang, C. C.; Hriljac, J. A.; Anderson, P. A. Chemical Control of Thermal Expansion in Cation-Exchanged Zeolite A. Chem. Mater. 2014, 26, 1561-1566.

(40) Leardini, L.; Quartieri, S.; Vezzalini, G.; Arletti, R. Thermal behaviour of siliceous faujasite: Further structural interpretation of negative thermal expansion. Micropor. Mesopor. Mater. 2015, 202, 226-233.

(41) Attfield, M. P. Strong negative thermal expansion in siliceous faujasite. Chem. Commun. 1998, 601-602.

(42) Woodcock, D. A.; Lightfoot, P.; Villaescusa, L. A.; Díaz-Cabañas, M.-J.; Camblor, M. A.; Engberg, D. Negative Thermal Expansion in the Siliceous Zeolites Chabazite and ITQ-4: A Neutron Powder Diffraction Study. Chem. Mater. 1999, $11,2508-2514$. 
(43) Woodcock, D. A.; Lightfoot, P.; Wright, P. A.; Villaescusa, L. A.; Camblor, M. A. Strong negative thermal expansion in the siliceous zeolites ITQ-1, ITQ-3 and SSZ-23. J. Mater. Chem. 1999, 9, 349-351.

(44) Villaescusa, L. A.; Lightfoot, P.; Teat, S. J.; Morris, R. E. Variable-Temperature Microcrystal X-ray Diffraction Studies of Negative Thermal Expansion in the Pure Silica Zeolite IFR. J. Am. Chem. Soc. 2001, 123, 5453-5459.

(45) Pryde, A. K.; Hammonds, K. D.; Dove, M. T.; Heine, V.; Gale, J. D.; Warren, M. C. Rigid unit modes and the negative thermal expansion in $\mathrm{ZrW}_{2} \mathrm{O}_{8}$. Phase Transitions 1997, 61, 141-153.

(46) Tucker, M. G.; Goodwin, A. L.; Dove, M. T.; Keen, D. A.; Wells, S. A.; Evans, J. S. O. Negative Thermal Expansion in $\mathrm{ZrW}_{2} \mathrm{O}_{8}$ : Mechanisms, Rigid Unit Modes, and Neutron Total Scattering. Phys. Rev. Lett. 2005, 95, 90.

(47) Tao, J.; Sleight, A. The role of rigid unit modes in negative thermal expansion. J. Solid State Chem. 2003, 173, 442-448.

(48) Tschaufeser, P.; Parker, S. C. Thermal Expansion Behavior of Zeolites and $\mathrm{AlPO}_{4} \mathrm{~s} . J$. Phys. chem. 1995, 99, 10609-10615.

(49) Park, S.; Kunstleve, R.-W. G.; Graetsch, H.; Gies, H. Studies in Surface Science and Catalysis; Elsevier, 1997; pp 1989-1994.

(50) Barrera, G. D.; Bruno, J. A. O.; Barron, T. H. K.; Allan, N. L. Negative thermal expansion. J. Phys.: Condens. Matter 2005, 17, R217-R252.

(51) Liu, Z.; Gao, Q.; Chen, J.; Deng, J.; Lin, K.; Xing, X. Negative thermal expansion in molecular materials. Chem. Commun. 2018, 54, 5164-5176.

(52) Miller, W.; Smith, C. W.; Mackenzie, D. S.; Evans, K. E. Negative thermal expansion: a review. J. Mater. Sci. 2009, 44, 5441-5451. 
(53) Bieniok, A.; Hammonds, K. D. Rigid unit modes and the phase transition and structural distortions of zeolite rho. Micropor. Mesopor. Mater. 1998, 25, 193-200.

(54) Grüneisen, E. Theorie des festen Zustandes einatomiger Elemente. Annalen der Physik $1912,344,257-306$.

(55) Vieillard, P. A predictive model for the entropies and heat capacities of zeolites. Eur. J. Mineral. 2010, 22, 823-836.

(56) Chibani, S.; Coudert, F.-X. Systematic exploration of the mechanical properties of 13621 inorganic compounds. Chem. Sci. 2019, 10, 8589-8599.

(57) Gaillac, R.; Chibani, S.; Coudert, F.-X. Speeding Up Discovery of Auxetic Zeolite Frameworks by Machine Learning. Chem. Mater. 2020, 32, 2653-2663.

(58) Siddorn, M.; Coudert, F.-X.; Evans, K. E.; Marmier, A. A systematic typology for negative Poisson's ratio materials and the prediction of complete auxeticity in pure silica zeolite JST. Phys. Chem. Chem. Phys. 2015, 17, 17927-17933.

(59) Dagdelen, J.; Montoya, J.; de Jong, M.; Persson, K. Computational prediction of new auxetic materials. Nature Commun. 2017, 8, 124.

(60) de Jong, M.; Chen, W.; Angsten, T.; Jain, A.; Notestine, R.; Gamst, A.; Sluiter, M.; Krishna Ande, C.; van der Zwaag, S.; Plata, J. J.; Toher, C.; Curtarolo, S.; Ceder, G.; Persson, K. A.; Asta, M. Charting the complete elastic properties of inorganic crystalline compounds. Sci. Data 2015, 2, 345.

(61) Coasne, B.; Haines, J.; Levelut, C.; Cambon, O.; Santoro, M.; Gorelli, F.; Garbarino, G. Enhanced mechanical strength of zeolites by adsorption of guest molecules. Phys. Chem. Chem. Phys. 2011, 13, 20096. 
(62) Ortiz, A. U.; Boutin, A.; Fuchs, A. H.; Coudert, F.-X. Investigating the PressureInduced Amorphization of Zeolitic Imidazolate Framework ZIF-8: Mechanical Instability Due to Shear Mode Softening. J. Phys. Chem. Lett. 2013, 4, 1861-1865.

(63) Chapman, K. W.; Chupas, P. J. Pressure Enhancement of Negative Thermal Expansion Behavior and Induced Framework Softening in Zinc Cyanide. J. Am. Chem. Soc. 2007, 129, 10090-10091.

(64) Fang, H.; Phillips, A. E.; Dove, M. T.; Tucker, M. G.; Goodwin, A. L. Temperaturedependent pressure-induced softening in $\mathrm{Zn}(\mathrm{CN})_{2}$. Phys. Rev. B 2013, 88.

(65) Fang, H.; Dove, M. T. A phenomenological expression to describe the temperature dependence of pressure-induced softening in negative thermal expansion materials. $J$. Phys. Condens. Matter 2014, 26, 115402.

(66) Fang, H.; Dove, M. T. Pressure-induced softening as a common feature of framework structures with negative thermal expansion. Phys. Rev. B 2013, 87.

(67) Fang, H.; Dove, M. T.; Phillips, A. E. Common origin of negative thermal expansion and other exotic properties in ceramic and hybrid materials. Phys. Rev. B 2014, 89.

(68) Tesson, S.; Louisfrema, W.; Salanne, M.; Boutin, A.; Rotenberg, B.; Marry, V. Classical Polarizable Force Field To Study Dry Charged Clays and Zeolites. J. Phys. Chem. C 2017, 121, 9833-9846.

(69) Combariza, A. F.; Gomez, D. A.; Sastre, G. Simulating the properties of small pore silicazeolites using interatomic potentials. Chem. Soc. Rev. 2013, 42, 114-127.

(70) Shi, H.; Migues, A. N.; Auerbach, S. M. Ab initio and classical simulations of the temperature dependence of zeolite pore sizes. Green Chem. 2014, 16, 875-884.

(71) Bureekaew, S.; Amirjalayer, S.; Tafipolsky, M.; Spickermann, C.; Roy, T. K.; Schmid, R. 
MOF-FF - A flexible first-principles derived force field for metal-organic frameworks. Phys. Status Solidi B 2013, 250, 1128-1141.

(72) Schmidt, J. R.; Yu, K.; McDaniel, J. G. Transferable Next-Generation Force Fields from Simple Liquids to Complex Materials. Acc. Chem. Res. 2015, 48, 548-556.

(73) Li, Y.; Li, H.; Pickard, F. C.; Narayanan, B.; Sen, F. G.; Chan, M. K. Y.; Sankaranarayanan, S. K. R. S.; Brooks, B. R.; Roux, B. Machine Learning Force Field Parameters from Ab Initio Data. J. Chem. Theory Comput. 2017, 13, 4492-4503. 


\section{Graphical TOC Entry}

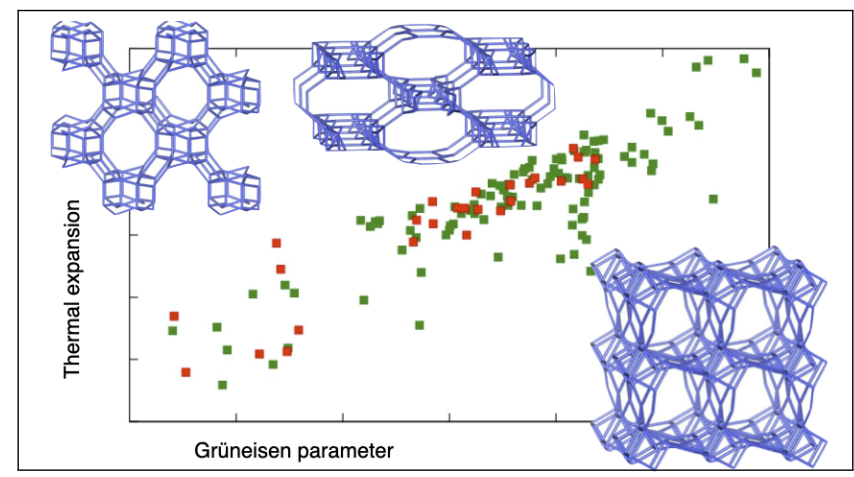

